\title{
Electronic crosstalk impact assessment in the Terra MODIS mid-wave infrared bands
}

\author{
Truman Wilson ${ }^{\mathrm{a}}$, Ashish Shrestha ${ }^{\mathrm{a}}$, and Xiaoxiong Xiong ${ }^{\mathrm{b}}$

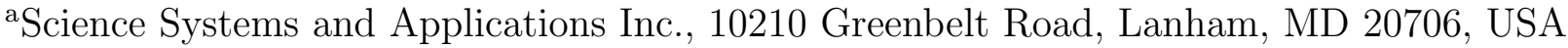 \\ ${ }^{\mathrm{b}}$ Sciences and Exploration Directorate, NASA/GSFC, Greenbelt, MD 20771, USA
}

\begin{abstract}
The Moderate Resolution Imaging Spectroradiometer (MODIS) on board the Terra spacecraft is one of the key instruments in NASA's Earth Observing System. Since 2000, MODIS has collected continuous data in 36 spectral bands ranging in wavelength between $0.4 \mu \mathrm{m}$ and $14.2 \mu \mathrm{m}$. Since before launch, signal contamination in the form of electronic crosstalk has been observed in many of the MODIS thermal emissive bands, particularly for bands $27-30$, a correction for which has been applied to the current Collection 6 algorithm. The mid-wave infrared bands in Terra MODIS, $20-25$, also show signs of electronic crosstalk contamination, which can be seen clearly during observations of the Moon. In this paper, we'll present an impact assessment of electronic crosstalk on the mid-wave infrared bands in Terra MODIS. We will also derive correction coefficients from the lunar observations, which can be applied to correct the calibrated radiance in the MODIS Level-1B product. We will provide an analysis of these results and potential improvements to the MODIS Level-1B product.
\end{abstract}

Keywords: MODIS, crosstalk, radiometric improvements, contamination, calibration, striping, thermal emissive bands (TEBs)

\section{INTRODUCTION}

The Earth-Observing System (EOS) Terra spacecraft was launched on December 18, 1999 with a suite of remote sensing instruments. ${ }^{1}$ Among these instruments, the Moderate Resolution Imaging Spectroradiometer (MODIS) obtains nearly continuous Earth-view imagery using 36 spectral bands which range in wavelength from $0.4-14.2$ $\mu \mathrm{m} .{ }^{2}$ The MODIS instrument is a whisk-broom, scanning radiometer, with a double-sided, rotating scan mirror which is used to direct light toward the MODIS detectors. Each MODIS band consists of a linear array of detectors which produce a continuous image through timed sampling of the detectors. For each Earth-view scan, MODIS acquires an image that is $10 \mathrm{~km}$ wide in the track direction at nadir by $2330 \mathrm{~km}$ long in the scan direction. At nadir, MODIS bands 1 and 2 have a resolution of $250 \mathrm{~m}$, bands $3-7$ have a resolution of $500 \mathrm{~m}$, and bands $8-36$ have a resolution of $1 \mathrm{~km}$. Away from nadir, the footprint of each pixel on the Earth's surface increases with the angle of the view away from nadir.

The MODIS bands are calibrated on-orbit using a set of onboard calibrators (OBC), such as the solar diffuser (SD) for calibrating the reflective solar bands (RSB) with its degradation measured by a SD stability monitor $(\mathrm{SDSM}){ }^{3}$ the blackbody (BB) for calibrating the thermal emissive bands (TEB) ${ }^{4}$ and the spectroradiometric calibration assembly (SRCA) for characterizing the spatial and spectral behavior of the detectors. ${ }^{5}$ MODIS also uses external targets such as selected Earth-view reference sites and scheduled observations of the Moon in order to characterize the scan mirror response versus scan angle (RVS) at angles of incidence on the scan mirror other than those of the OBCs. ${ }^{6,7}$

MODIS has four focal plane assemblies (FPA), the visible (VIS), near-infrared (NIR), short- and mid-wave infrared (SMIR), and the long-wave infrared (LWIR), which group the bands by wavelength. The SMIR and LWIR FPAs are cooled to a set-point temperature of $83 \mathrm{~K}$. A diagram of the SMIR FPA layout can be seen in Figure 1. This FPA consists of bands at both $500 \mathrm{~m}$ (bands $5-7$ ) and $1 \mathrm{~km}$ (bands $20-26$ ) resolutions, which are sampled by separate sets of electronics respectively. For each frame in a MODIS scan, each detector for a 


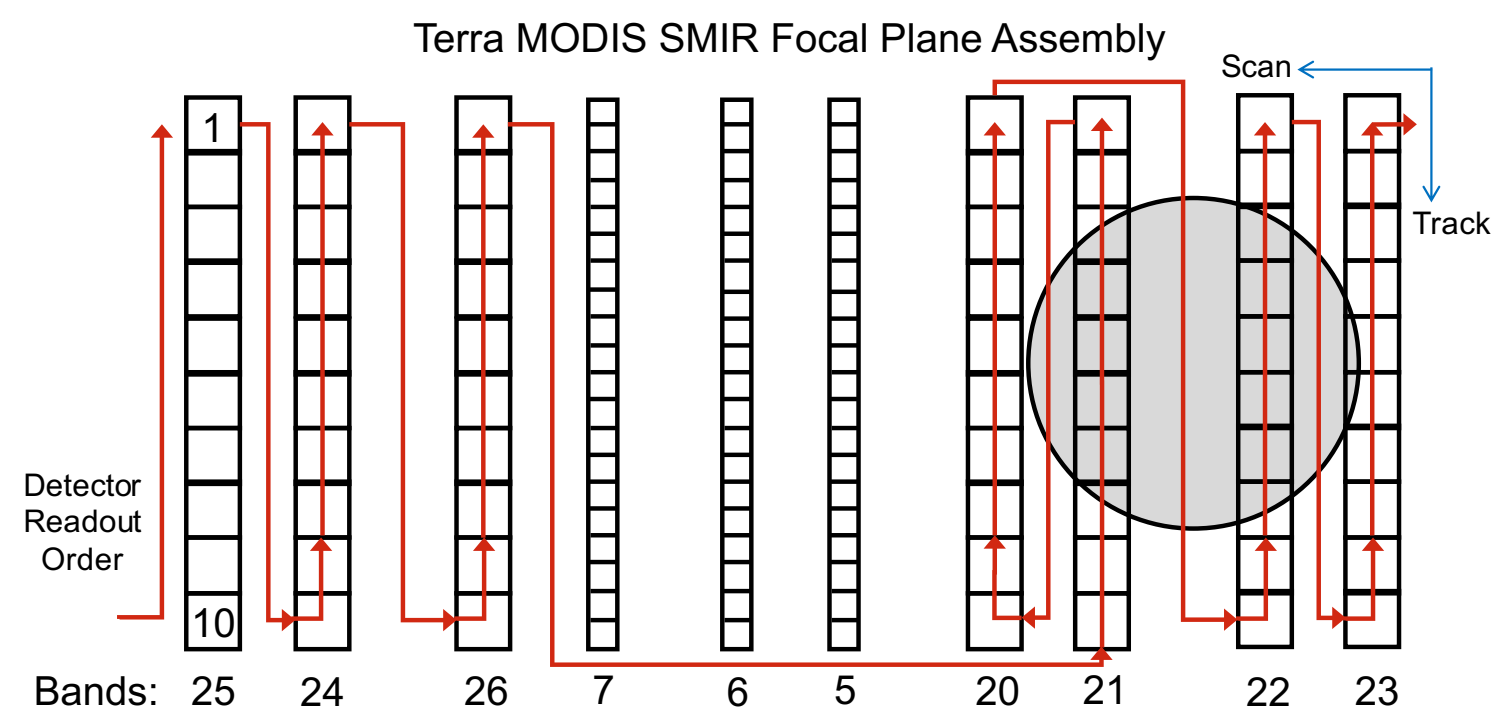

Figure 1. Terra MODIS focal plane assembly layout for the short- and mid-wave infrared bands. The 1-km resolution bands, $20-26$, have 10 detectors and are sampled using the same electronics. The order of the electronic readout for each frame is shown by the red arrows. The detector order is shown in product order with detector 1 of each band at the upper part of the figure. Superimposed on this image is a representation of the size of the lunar image as focused on the FPA (grey circle).

group of bands is sampled in a specific order by the detector electronics. For the 1-km bands on the SMIR FPA, the order of the sampling of each frame is given by the red arrows in Figure 1.

Since launch, several of the MODIS TEBs have been impacted by signal contamination among the bands in the form of electronic crosstalk. ${ }^{8-12}$ This issue has been studied extensively for the photovoltaic bands on the LWIR FPA, bands 27-30, where the contamination levels have increased significantly as the mission progressed. ${ }^{10,12}$ For these bands in Terra MODIS, the contamination was characterized and corrected by using observations of the moon, where the moon provides a small, bright target against the background of space which allows for the contaminating signal to be easily identified. ${ }^{12}$

For the mid-wave infrared (MWIR) bands, lunar observations also show signal contamination away from peak lunar signal. As the scan mirror rotates, the lunar image moves across the FPA in the scan direction. Since the bands are spatially separated on the FPA, as seen in Figure 1, each band observes the same part of the Moon at a slightly different time. Also, because of the large distance between the instrument and the Moon, the lunar image width is approximately 6 MODIS 1-km pixels. This means that as some bands observe the Moon, others will be viewing deep space. If there is electronic crosstalk contamination coming from one of the bands that is observing the moon, this effect can be observed directly as an anomalous signal that is either above or below the background level, away from where we would expect to observe the moon for a particular detector.

For a selected MODIS detector, we can plot the background-subtracted, measured lunar signal versus scan and frame number as seen in Figure 2. Because of the oversampling effect, the Moon can be observed for many consecutive scans. ${ }^{13}$ For most TEBs, the lunar signal saturates at a digital count of 4095 before background subtraction. Since the signal contamination occurs before the analog-to-digital conversion, in order to accurately model the contaminating signal, this saturation needs to be corrected. To do this, we replace the saturated pixels using a reference signal from band 31, which does not saturate during observations of the moon for Terra MODIS. Here, we use the ratio of unsaturated pixels from our target band and band 31 to correct the saturation, as seen in Figure 2.

Further author information: (Send correspondence to T.W.)

T.W.: E-mail: truman.wilson@ssaihq.com, Telephone: 13018672120 
While the lunar signal at full scale does not show any significant anomalies, if we focus on the low signal behavior (right plot of Figure 2), we can see that there are several anomalous peaks outside of the main lunar signal, which is cut off at the top of this plot. These anomalous peaks represent the crosstalk contamination, which can be both positive and negative with respect to the background level. In order to determine the source of the contaminating signal, we can plot scaled versions of the lunar signal from the other MWIR bands, which share the same sampling electronics. In Figure 3, we show the signal contamination alignment for two selected detectors among the MWIR bands. In the left plot, we show the contamination for band 21 detector 1. In this case, the contaminating signal comes from a single detector, band 20 detector 10, which is plotted to show the alignment with the contaminating signal. In the right plot, we show the contamination for band 23 detector 10. This detector receives contaminating signal from all of the detectors of multiple sending bands, which are listed in the plot. For this detector, we also observe a significant component of the contaminating signal comes from detectors within the same band, which was also observed for the LWIR bands in Terra MODIS. ${ }^{12}$ Also, we noticed that for band 22 detector 8 and band 23 detector 10, there was a component of contamination at higher frame number relative to band 23 that did not align well with any potential sending signal from the SMIR FPA, as band 23 is one of the outer bands on the SMIR FPA (Figure 1). As of this writing, the source of this contamination is unclear, however, it does not seem to affect our ability to derive the correction coefficient for band 23 sending. We also noticed that there was the potential for some sending contribution from bands $5-7$ for this detector. However, when we allowed sending from these bands in our algorithm, the correction introduced significant anomalies to the Earth-view data, and therefore these bands are removed from consideration.
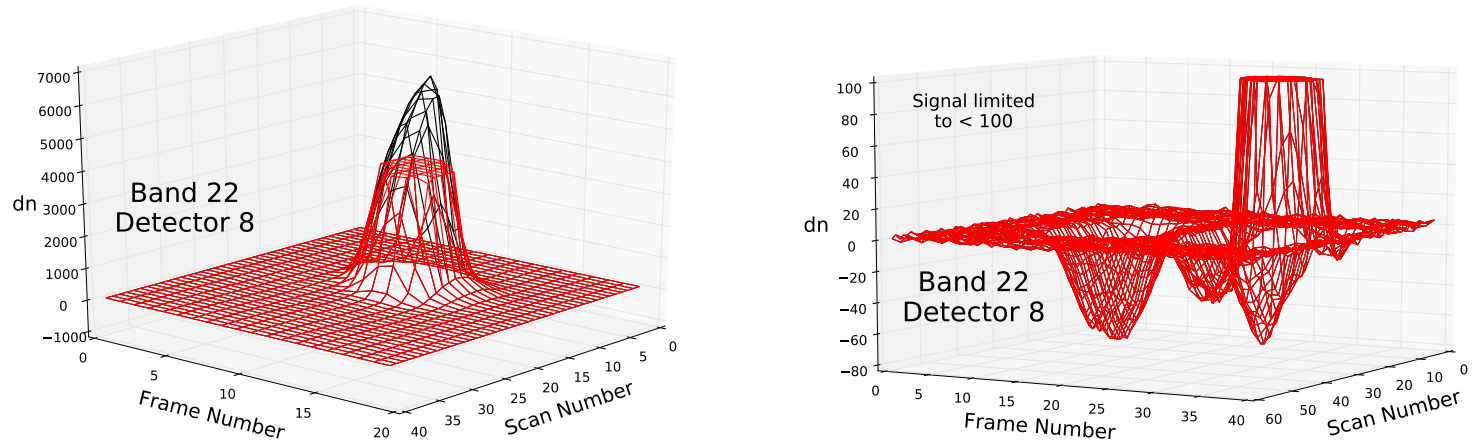

Figure 2. (left) Example of single detector lunar data for band 22 detector 8 before (red) and after (black) the saturation correction is applied. (right) Example contamination data at low signal around the main lunar signal. The data for these plots was from the lunar observation on May 26, 2016.
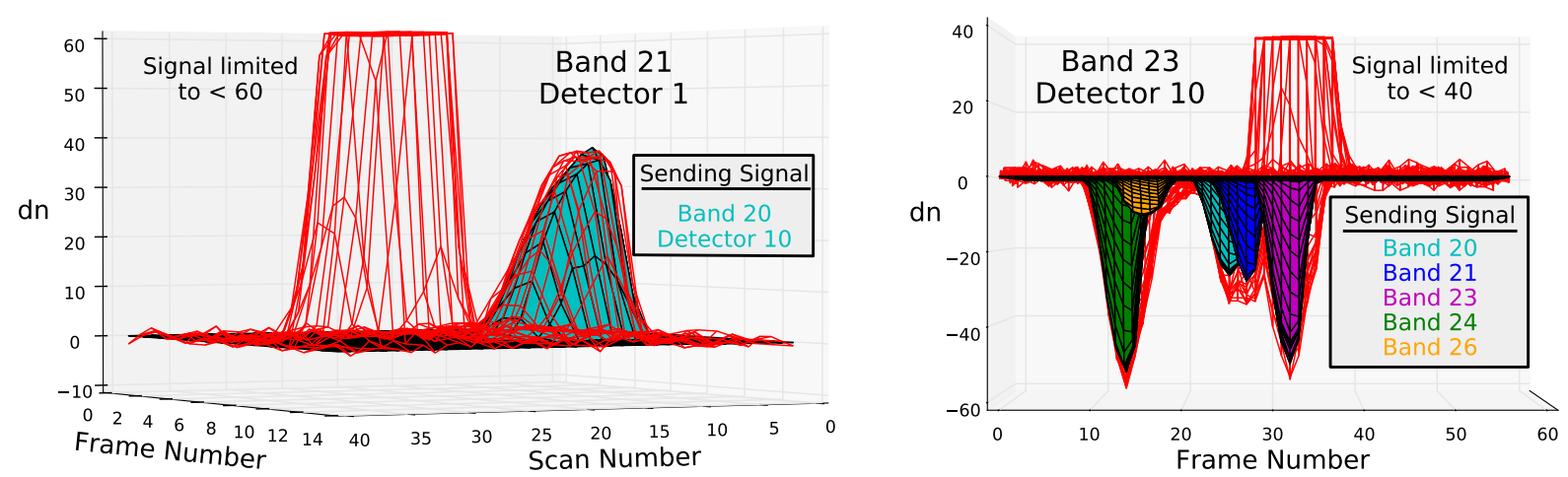

Figure 3. Example sending signal alignments with the appropriate scan and frame offsets for the sending signals. (left) Band 21 detector 1 receiving, with contamination sent by band 20 detector 10. (right) Band 23 detector 10 receiving, with sending signals from bands 20, 21, 23, 24, and 26. Bands 22 and 25 do not contribute significantly to the contamination for this detector. The sending signals are shown after saturation correction for bands 20, 23, and 24, and are scaled to show their alignment with the signal contamination. 
With the source of the contaminating signals identified as the other bands on the SMIR FPA, we can now develop an algorithm to derive the appropriate correction coefficients from the lunar observations for each contaminated detector. With these coefficients, we can apply them to the Level-1B data and OBC to derive the corrected Earth-view scene radiance, and analyze its impact.

\section{CORRECTION ALGORITHM}

The lunar-based correction has been used extensively in previous attempts at electronic crosstalk correction for many MODIS bands. ${ }^{8-12}$ In each of these efforts, the contaminating signal has been assumed to be linearly proportional to the measured signal from the identified sending bands. Since electronic crosstalk affects the digital signal in each data sector, it will have an impact on background signal as well as the signal from any measured Earth-view or OBC scene. However, since the background contamination is at a nearly constant level, this contamination can be subtracted off with the rest of the background signal. Therefore, we can write the corrected, background-subtracted signal for the $i$ th detector as:

$$
d n_{i}(S, F)=d n_{i}^{*}(S, F)-\sum_{j} c_{i, j} \cdot d n_{j}^{*}\left(S, F+\Delta F_{j}\right)
$$

where the $d n^{*}$ represents the measured (contaminated) signal after background subtraction, $S$ and $F$ represent the scan and frame number of the image pixel, $i$ and $j$ represent the receiving detector and sending detector index respectively, $c_{i, j}$ is the crosstalk coefficient matrix, and $\Delta F_{j}$ is the frame offset for sending detector $j$. For the crosstalk correction among the MWIR bands, $i$ ranges between $1-60$ representing each of the ten detectors in bands $20-25$ in product order. The sending index, $j$, ranges between $1-70$ to include the sending contribution from band 26, which is a short-wave infrared band. The crosstalk contamination of band 26 is the subject of on-going work on the crosstalk correction of the short-wave infrared bands, and will not be presented in this work.

To solve for the elements of $c_{i, j}$, we perform a least-squares minimization calculation in which we compare the corrected lunar signal to a reference signal for each pixel outside of the main lunar signal, which is defined as pixels in the reference signal with $d n<150$. For consistency with the crosstalk correction performed for bands $27-30,{ }^{12}$ we choose band 31 as a reference signal, as it shows no signs of electronic crosstalk and will help us to model the expected behavior of our lunar observations at low-signal levels near the edge of the moon, where the signal from the moon does not cut off sharply. However, for band 21, an exception is made because the low overall signal level allows us to use the background level as our reference signal. Also, for most detectors, the contamination occurs far enough away from the main lunar signal in both scan and frame number that the reference signal does not play a significant role. So, we can solve for the crosstalk coefficients, $c_{i, j}$, by minimizing the value of $\eta_{i}^{2}$ in the following equation for each contaminated detector, $i$.

$$
\eta_{i}^{2}=\sum_{S, F}\left(d n_{i}^{*}(S, F)-d n_{r e f, i}(S, F)-\sum_{j} c_{i, j} \cdot d n_{j}^{*}\left(S, F+\Delta F_{j}\right)\right)^{2}
$$

The coefficient matrix, $c_{i, j}$, is set up so that each sending and receiving detector is paired with a individual coefficient value. However, in practice, we've found that significant overlap between the individual sending signals between neighboring detectors makes it difficult separate the contributions of each sending detector to the contamination. To simplify our analysis, we start by fixing all of the coefficients for each detector within a given sending band to the same value. Then, we analyze the contaminated signal for each detector in order to identify sending anomalies that align with specific sending detectors, and we allow those coefficients to be derived independently from the rest of the band. A common case for individual detector contamination occurs between detector 10 of a band that is beginning its electronic sampling and detector 1 of a band that has just finished its electronic sampling, as illustrated in Figure 1 and shown in Table 1. This contamination appears as an individual peak on the detector 1 signal that precisely aligns with the detector 10 sending signal from the next sampled band, as can be seen in Figure 3 and in several examples in Figure 4. This type of contamination is also observed for many other bands in both Aqua and Terra MODIS. ${ }^{11,12}$ 
Table 1. A table listing the sending and receiving detectors related to the electronic sampling error between successively sampled bands. The detectors are listed in product order.

\begin{tabular}{cc}
\hline \hline Sending Detector & Receiving Detector \\
\hline Band 20, Detector 10 & Band 21, Detector 1 \\
Band 21, Detector 10 & Band 26, Detector 1 \\
Band 22, Detector 10 & Band 20, Detector 1 \\
Band 23, Detector 10 & Band 22, Detector 1 \\
Band 24, Detector 10 & Band 25, Detector 1 \\
Band 25, Detector 10 & Band 23, Detector 1 \\
Band 26, Detector 10 & Band 24, Detector 1 \\
\hline
\end{tabular}

Aside from the electronic sampling error, there were other instances of individual sending detectors that needed coefficients to be separated from the band-average. This includes band 25 detector 1 , sending to band 23 detector 1, which can be seen as the smaller positive peak in the band 23 detector 1 signal in Figure 4 (the larger peak is from band 25 detector 10). For the correction to the detectors in bands 22 and 23 , we also found that the lunar correction was improved if selected sets of detectors from band 24 were isolated from the rest of the band, however, the impact of this on the overall band average is small.

An example of the crosstalk correction on the lunar observation data can be seen in Figure 4 for a selected detector in each band. For most bands, the most significant contribution to the signal contamination occurs in detector 1 of each band from the electronic sampling error. However, in the case of band 22 detector 8 (right plot of the top row in Figure 4) and band 23 detector 10 (right plot in Figure 3), there is a significant contribution to the contamination from the other MWIR bands. This signal contamination is typically negative relative to the background signal.
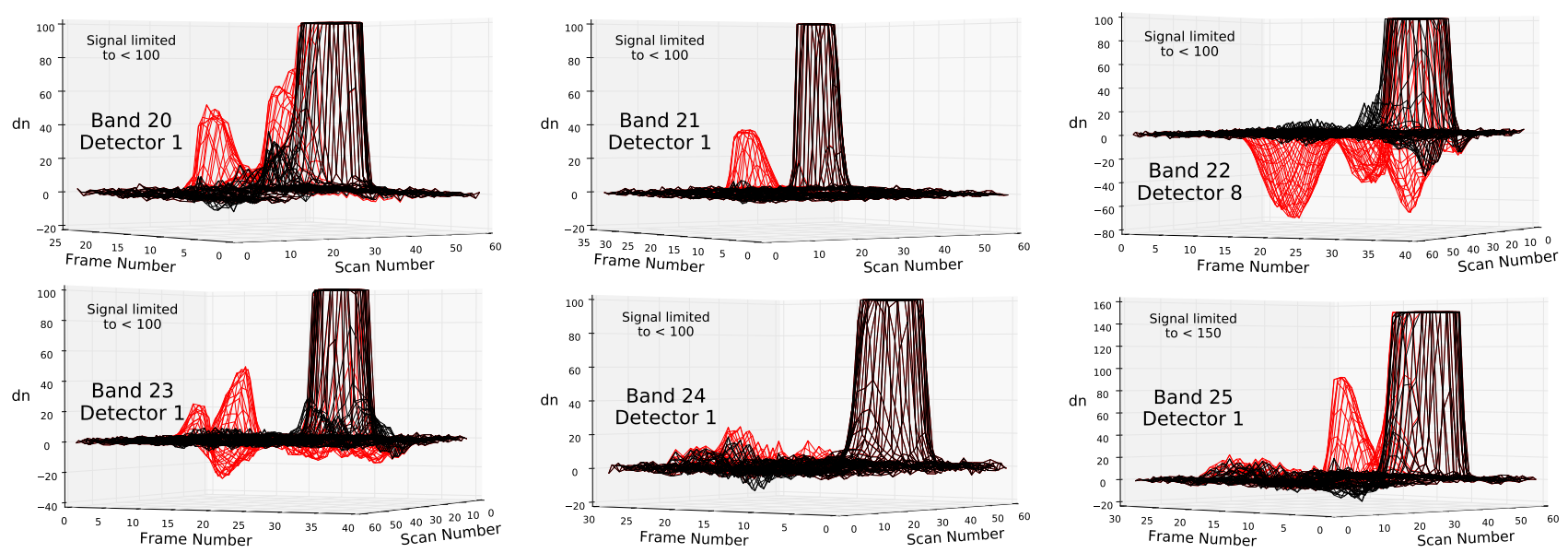

Figure 4. Example corrections of the lunar signal for one detector from each band. The red data shows the lunar signal before the correction is applied and the black data shows the lunar signal after the correction is applied. For most of the MWIR bands, the most significant contamination in the lunar signal occurs for detector 1 . The data shown here is from the lunar observation on May 26, 2016.

Using this algorithm, we can derive crosstalk coefficients for each detector using lunar observations throughout the mission. In the first two years of the mission, the electronics configuration was changed multiple times, which changed the nature of the crosstalk contamination significantly. Therefore, for this work, we only analyze data for lunar observations starting in September 2001. In Figure 5, we show the crosstalk coefficients for the detector 10 to detector 1 sampling anomaly. For most bands, the coefficients are at a nearly constant level, at or around 0.01 throughout the entire mission. For band 24 detector 1 , the band 26 detector 10 sending coefficient is much larger than that of the rest of the bands, even though the absolute level of contamination for this detector is smaller, as seen in Figure 4. This is because the signal for band 26 when viewing the Moon is much lower than 
that of the TEBs (except band 21), and translates to a larger coefficient value, even for lower absolute levels of contamination.

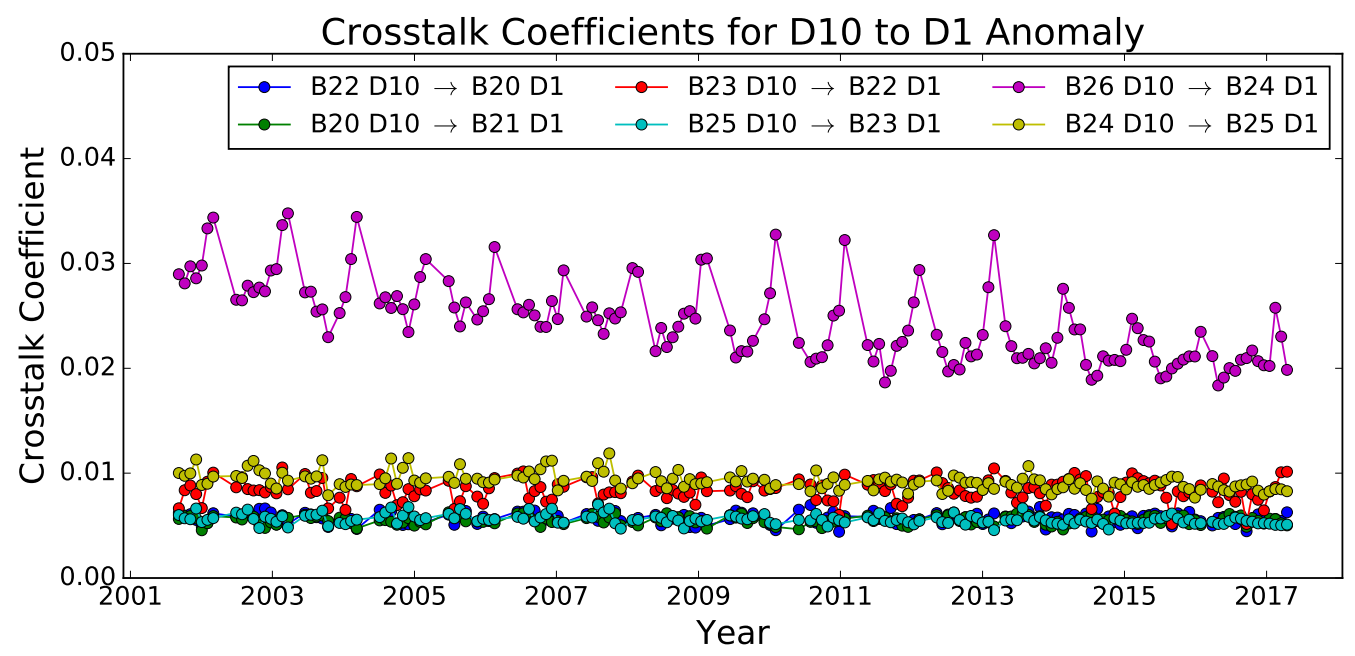

Figure 5. Trending crosstalk coefficients for detector 10 sending to detector 1 of successively sampled bands.

In Figure 6, we show the coefficient values for each sending band for band 22 detector 8 and band 23 detector 10 receiving, which show similar behavior for the coefficients for both bands. Notably, the magnitude of the coefficient values are largest for bands 21 and 26 sending. Similar to the band 26 coefficient for band 24 discussed previously, these two bands have the smallest measured signal when viewing the moon, particularly for band 21.
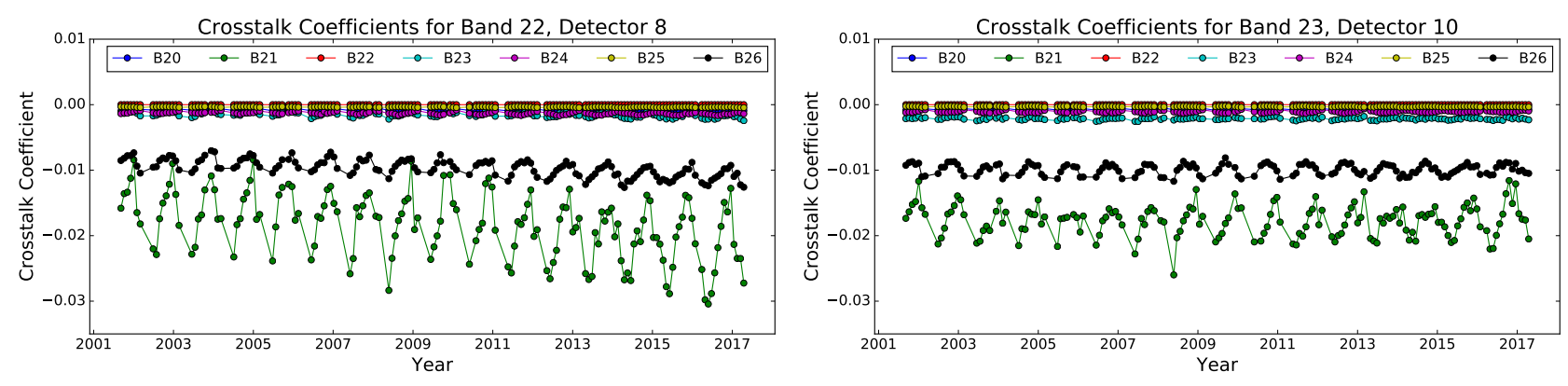

Figure 6. Example trending crosstalk coefficients. (left) Band 22 detector 8 receiving. (right) Band 23 detector 10 receiving.

The oscillating behavior of the bands 21 and 26 sending coefficients warrants closer inspection. The lunar signal for these bands is very small compared to the surrounding MWIR bands, and the derived coefficient values can be sensitive to the overlap of the signal of these bands and their neighbors. For band 21, the Earth-view signal is also small, as the gain of band 21 is set at a relatively low value for its use in fire detection. As of this writing, we have not been able to identify a noticeable impact of the oscillations of the band 21 coefficients in the L1B correction using these coefficients. For band 26, the signal can be large over highly reflective ice clouds. Incorrectly deriving the correction coefficients from the Moon can have a large impact over these scenes, as will be discussed in Section 4.

\section{APPLICATION OF THE CORRECTION COEFFICIENTS}

Since electronic crosstalk occurs in the MODIS instrument at the raw signal level, it is necessary to apply the crosstalk correction to all of the data sectors, which include the OBCs and Earth-view sectors, using the same method as in Equation 1. The calibrated Earth-view scene radiance, $L_{E V}$, for a given pixel is given by: 


$$
L_{E V}=\frac{1}{\operatorname{RVS}_{E V}}\left(a_{0}+b_{1} d n_{E V}+a_{2} d n_{E V}^{2}-\left(\operatorname{RVS}_{S V}-\mathrm{RVS}_{E V}\right) L_{S M}\right)
$$

Here, $a_{0}, b_{1}$, and $a_{2}$ are the offset, linear and quadratic gain coefficients respectively, $d n_{E V}$ is the backgroundsubtracted digital signal of the Earth-view pixel, RVS is the response versus scan angle for the Earth-view pixel (EV) and space-view (SV), and $L_{S M}$ is the background radiance associated with the scan-mirror thermal emission. In order to appropriately correct the Earth-view data, we first apply the crosstalk correction to the calibration data in order to derive corrected gain coefficients. The non-linear gain parameters, $a_{0}$ and $a_{2}$, are calculated during regularly scheduled BB warm-up/cool-down events, where the BB temperature is changed from its nominal set point in order to determine the non-linear response of the detectors. ${ }^{4}$ For these non-linear gain parameters, the impact of the correction is minimal, and we observe almost no change compared to the uncorrected data.

The linear gain term, $b_{1}$, is calculated on a scan-by-scan basis, using the average of each view of the $\mathrm{BB}$ source of the surrounding 40 scans for the same scan-mirror side. When applying the crosstalk correction to the scan-by-scan BB data, for most detectors, the value of $b_{1}$ changes by less than $1 \%$. For band 22 detector 8 and band 23 detector 10, there is a larger overall shift in the value of $b_{1}$ by $\sim 3 \%$. Since the level of contamination is consistent throughout the mission, the crosstalk correction introduces a nearly constant bias to the uncorrected value of the gain, and does not introduce any drift.

With the gain coefficients corrected, we can then apply the crosstalk correction to the Earth-view signal $d n_{E V}$, and analyze the impact of the correction on the L1B calibrated radiance product. For most detectors, the impact is minimal. However, there are a few detectors where the correction provides an overall improvement to the imagery for some scenes, which will be discussed in the following sections.

\section{CORRECTING EARTH-VIEW DATA AT LOW RADIANCE}

For the crosstalk contamination from sending band 26, the impact on the Earth-view imagery is greatest for daytime scenes over ice clouds, where the measured signal for TEBs is low due to the low scene temperature, and the signal for RSBs is high due to the high reflectivity of ice clouds. When applying the crosstalk correction using coefficients derived from lunar observations, we noticed that the correction for low-radiance scenes was inconsistent for band 22 detector 8, band 23 detector 10, and band 24 detector 1 . For the detectors in bands 22 and 23, the Earth-view imagery was typically over-corrected as seen in Figure 7 for band 23. For band 24, the oscillations in our derived coefficient values result in an appropriate correction during some parts of the mission and under correction for many other parts of the mission.

These results strongly suggest that the lunar-based correction for band 26 sending is insufficient. It is likely that a combination of the relatively low signal size during lunar observations for band 26 relative to other TEBs and its proximity to the high signal levels of band 24 make determining the band 26 coefficient difficult. For the contamination in bands 22 and 23, the neighboring contaminating signal from band 24 is at least 5 times greater, and overlaps with parts of the band 26 signal, resulting in an over-estimate of the band 26 coefficient. For the contamination in band 24, this contaminating signal is, at times, difficult to distinguish from the tail of the lunar signal.

Since the impact of this low-radiance correction is only significant on a single receiving detector in bands $22-24$ and because we know that the sending contamination comes from band 26, we can use the Earthview imagery in order to derive a correction coefficient from band 26 for these detectors. To do this, we can compare the histograms of calibrated radiance retrieval for each detector for a single band for daytime scenes with a significant fraction of ice clouds (greater than 20\%), where the band 26 contamination is expected to be high. To determine the ice cloud fraction, we use the MODIS Cloud Particle Phase (MOD06_L2) ${ }^{14}$ and Cloud Mask products (MOD35_L2). ${ }^{15}$ The NASA Level-1A (raw digital counts) and Level-1B (calibrated radiance) data products are split into data granules covering a 5-minute time range. These granules consist of over 200 individual scans of 1354 frames each. This means that for each detector there are over $2.7 \cdot 10^{5}$ individual radiance retrievals per granule. For this many retrievals over a large scene, we expect that each detector's radiance retrieval histogram will be nearly the same. 

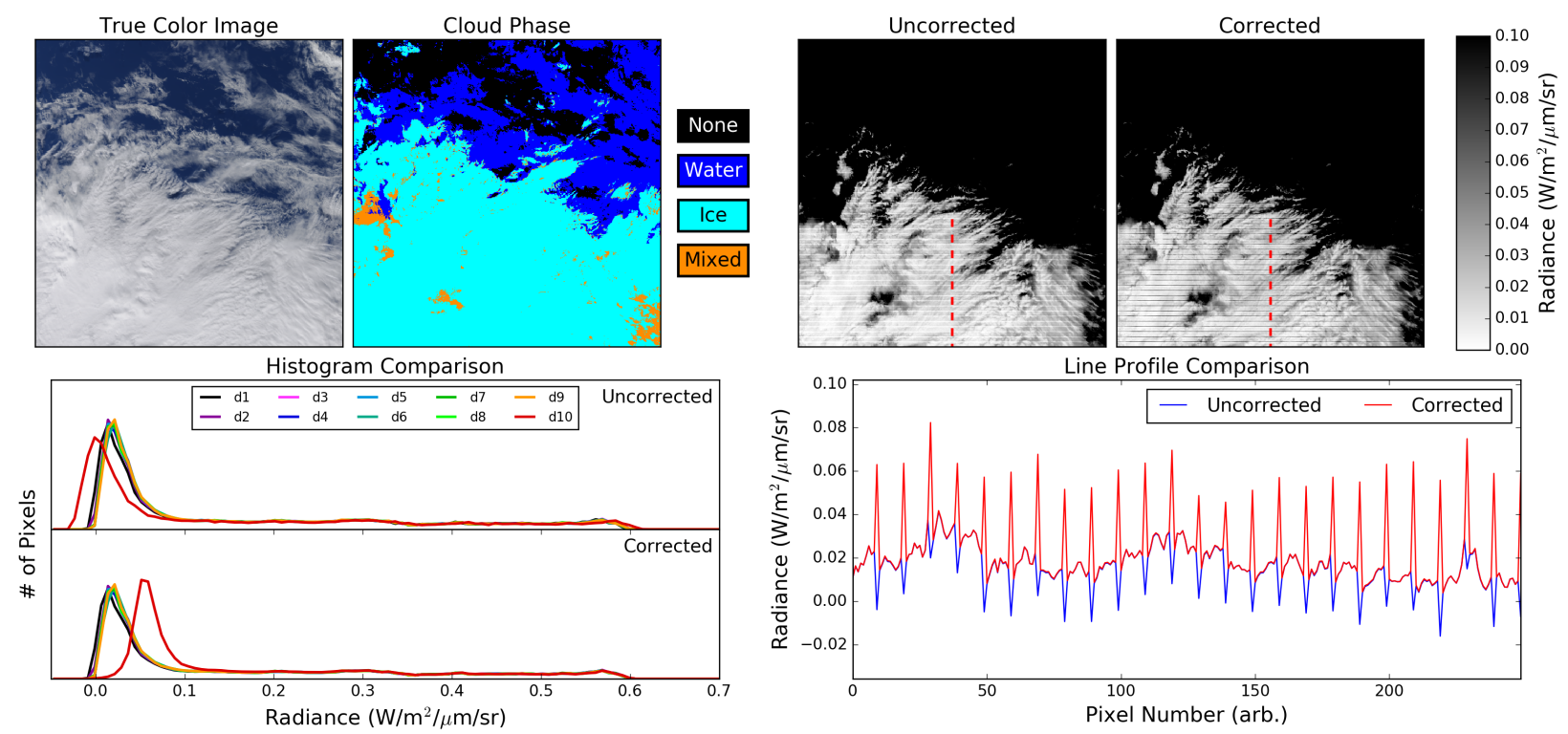

Figure 7. An example of the over-correction at low radiance for the crosstalk contamination of band 23 detector 10 for a selected granule on July 28, 2002. (top) A true color image, data from the Cloud Particle Phase product, uncorrected and crosstalk corrected images from left-to-right. The Cloud Particle Phase data shows that the scene has a significant fraction of ice clouds in the lower half of the image, where we expect significant contamination from band 26. (bottom) The corrected and uncorrected histograms of detector radiance retrieval (bottom left) using coefficients derived from lunar data, and a line profile comparison (bottom right), indicated by the red lines in the upper figures. The lunar-based correction over-corrects this low-radiance data.

To determine the impact of the band 26 contribution to the crosstalk contamination, we take our Level-1A data and apply the crosstalk correction for each detector using coefficients derived from the Moon using Equation 1, with the exception of the band 26 coefficients, which are set to zero. We then calculate the calibrated radiance from this corrected Level-1A data, creating an effective Level-1B product. From the calibrated data, we can calculate the histograms of the radiance retrieval for each detector, which can be seen in Figure 7 for band 23 . For the affected detectors, at low radiance, the histograms show a significant difference, which can also be seen in images from these bands in the form of striping.

To make a comparison of the histograms, we can use the Kullback-Leibler (KL) divergence, ${ }^{16}$ which will allow us to determine the similarity between the histograms of our detector retrievals. Let the histogram for our affected detector be represented by $f(L)$, and our reference histogram, which we take to be the average histogram of the other 9 detectors within the band, be represented by $g(L)$. We can write the KL divergence, $D$, as:

$$
D=\sum_{i} f\left(L_{i}\right) \ln \frac{f\left(L_{i}\right)}{g\left(L_{i}\right)}
$$

Here, $L_{i}$ represents the radiance retrieval for bin $i$ in our histograms. If each distribution, $f(L)$ and $g(L)$, are normalized, then the KL divergence, $D$, can range in value between 0 and 1 , where a value of 0 represents similar distributions. Using this parameter, we can calculate the appropriate band 26 sending coefficient for our affected detectors by minimizing the value of $D$. After minimization, we can apply the calculated correction coefficient to the re-derive the L1B data as seen in Figure 8. Since our minimization process is based on comparisons of the histograms, the out-of-family response for detector 10 of band 23 in this example is almost perfectly restored in the histograms at low radiance as expected. Another test of the correction is in the vertical striping of the scene at low radiance, also seen in Figure 8. Using this new coefficient, we can see that the striping is now reduced, in contrast to the lunar-based correction seen in Figure 7. 

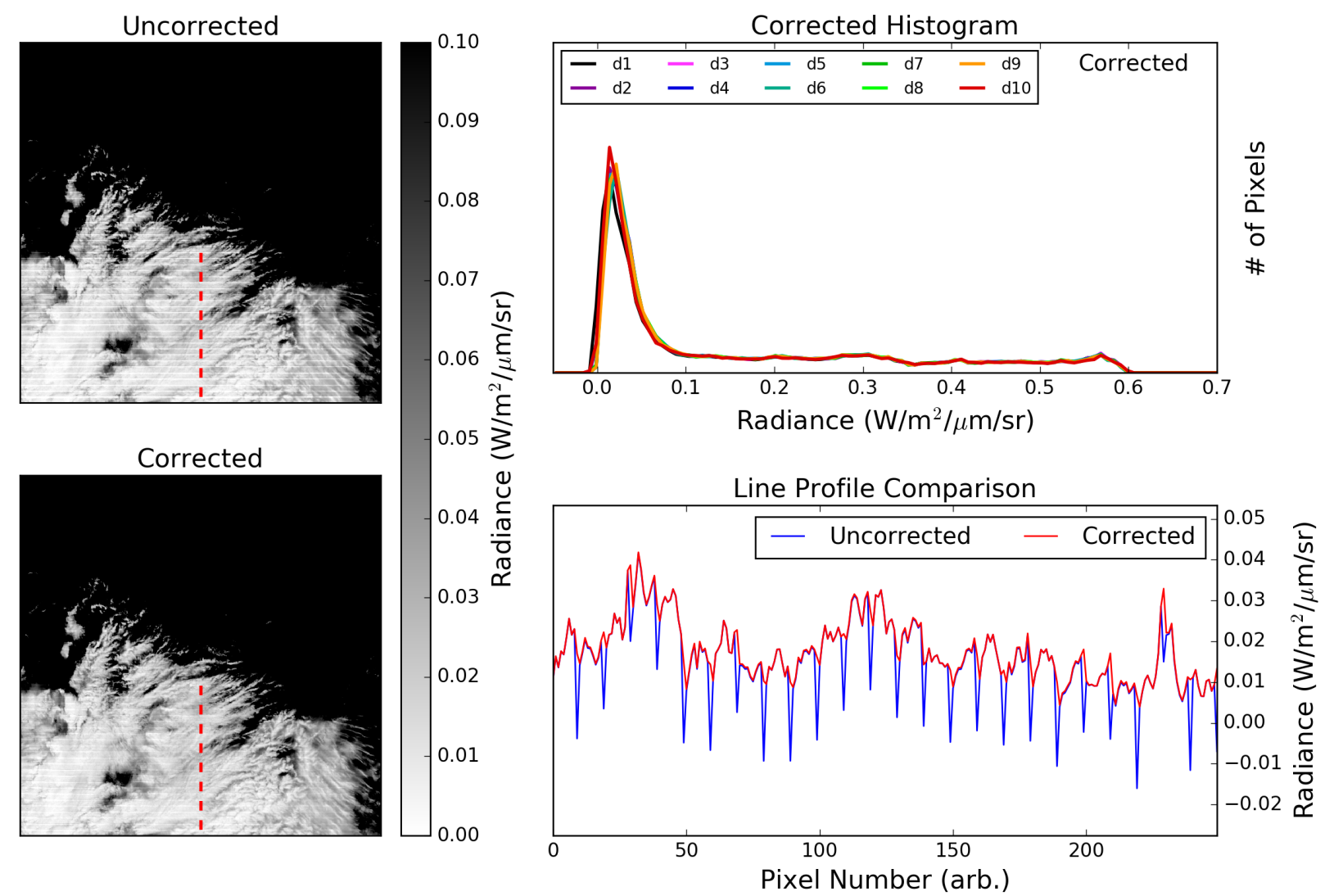

Figure 8. Corrected data using coefficients derived from Earth-view data for the same scene as in Figure 7. (left) A comparison of the uncorrected and newly corrected images. (top right) Corrected histogram of the detector radiance retrieval. (bottom right) A line profile comparison with the KL-based correction applied.

To re-derive the band 26 sending coefficients throughout the entire mission for bands $22-24$ detectors 8 , 10, and 1 respectively, we performed our KL minimization routine for selected granules on the day preceding each scheduled lunar observation since September 2001. On each day, we calculated the coefficient value for every daytime granule with a total ice cloud fraction greater than $20 \%$. We then calculated the average of the coefficients for each day using a 3 standard deviation outlier rejection. The results of this calculation can be seen in comparison to the derived lunar coefficients in Figure 9. For bands 22 and 23, we can see that the lunar-based derivation of the crosstalk coefficients from band 26 show a large bias towards more negative values and significant oscillations, which result in an over-correction of the low radiance data. The KL-based correction shows a lower magnitude value with significantly reduced oscillations which provides a much better correction of the low-radiance data throughout the mission. For band 24, we found that the oscillations were reduced, such that the value of the coefficient stays near the peak levels of the oscillation early in the mission. This new correction coefficient results in a more consistent correction throughout the mission, where the low points in the oscillations from the lunar coefficients under-corrected the data previously.

\section{CORRECTION OF THE LEVEL-1B DATA}

With the crosstalk correction coefficients from the lunar data and the KL method, we were able to analyze the impact of the correction on the MWIR Earth-scene data. Since late 2001, Terra MODIS has been operated in the same electronics configuration, and the level of contamination for the MWIR bands has been consistent throughout the rest of the mission. To analyze the impact of the correction throughout the mission, we applied the correction to various scenes for several dates for each MWIR band. We found that the applied correction produced consistent results throughout this time-period. 

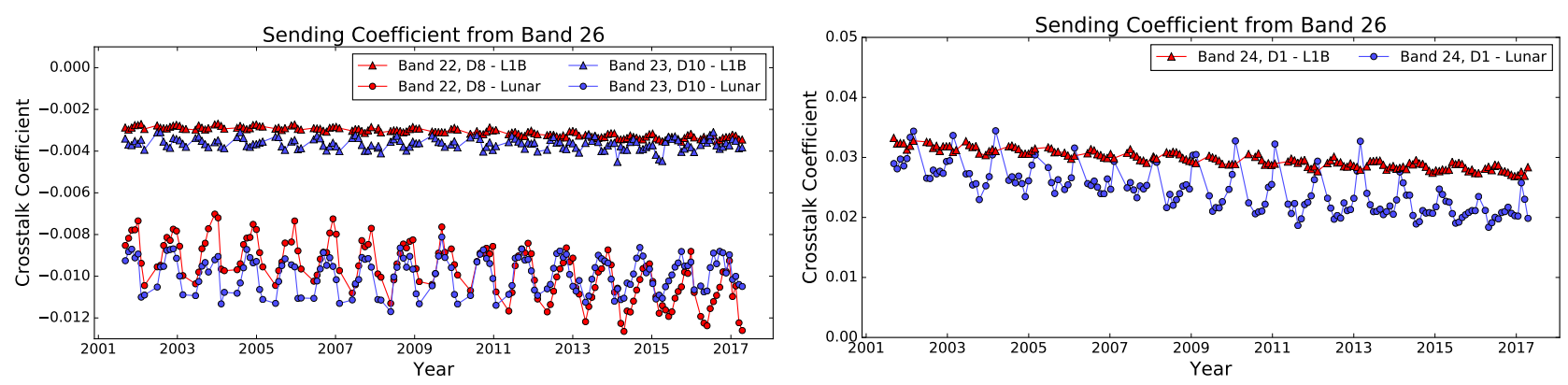

Figure 9. Comparison of the correction coefficients for band 26 sending using the L1B based correction and the lunarbased correction. (left) Band 22 detector 8 and band 23 detector 10 band-averaged coefficient for band 26 sending. (right) Band 24 detector 1 receiving from band 26 detector 10.

Although some level of contamination is seen in the lunar data for each band, the goal of this work is to determine whether or not a correction is necessary, and if our approach is able to provide this correction. To do this, we must look at the impact of the applied correction on the MODIS L1B Earth-view data. The largest effect is seen for bands $22-24$, particularly for daytime scenes over ice clouds as mentioned previously. However, there are other scenes which show the impact of the crosstalk contamination.

For band 22, the most significant impact of the crosstalk correction is observed on detector 8 , as the contamination levels in the lunar data are at a much larger level compared to the rest of the detectors (Figure 4). Analysis of the L1B data over a variety of scenes shows that the most contamination occurs over scenes with high fractions of ice clouds, related to the band 26 contamination, and over scenes of warm water where the contamination comes from other MWIR bands. An example correction for band 22 can be seen in Figure 10 over ocean. In this scene, we observe striping on detector 8 which is removed by the crosstalk correction. The histogram plots show a difference for detector 8 at both the low- and high-radiance parts of the scene, representing the different types of contamination present over ice clouds and ocean respectively. Using the combined coefficients from the Moon and the KL method allow us to correct both of these scenes. For scenes over water, the crosstalk correction is on the order of $\sim 2 \%$. Over ice clouds, the low radiance level results in a much higher fractional correction but has a similar magnitude to the high radiance correction as seen in the histograms of Figure 10 .

While the example for band 23 shown in Figures 7 and 8 shows the correction over ice cloud scenes, band 23 also shows image striping over warm water scenes, as seen in Figure 11. In this scene, striping is seen over the Mediterranean Sea near the Nile River delta for detectors 1 and 10. Over land, the band 23 signal is highly variable as seen on the right side of the line profile plot in Figure 11. Over water, the signal level is more consistent, and the crosstalk contamination can be clearly observed in the form of image striping. Applying the crosstalk correction has little impact over the land part of the scene, but removes the striping over the water, which is $\sim 2 \%$ of the total radiance level. In this particular scene, there are no ice clouds, so the correction has no impact on the low radiance part of the scene. However, studies of other scenes have shown that the correction works well for scenes with both water and ice cloud contamination.

For band 24, the crosstalk correction only has a substantial impact for detector 1 over ice clouds, where the sending signal from band 26 is high. The same issue has been studied extensively for this band and detector in Aqua MODIS ${ }^{11}$ with a successful correction applied using a lunar-based correction coefficient. In our case, we use the KL method for deriving coefficients for band 26, and the Moon for the rest of the sending bands. For detectors $2-10$, the impact of the correction is minimal. An example of the correction can be seen in Figure 12, for a scene of ice clouds over the ocean. Before the correction, striping can be seen in the band 24 image, but only over the cloudy part of the image, where the band 26 signal is high. The histogram before correction shows that the low radiance retrieval for detector 1 is out-of-family, and retrieves a much higher radiance than the other detectors within the band. After correction, the radiance retrieval is restored for detector one, and the striping in the image over the clouds is reduced. For this example, we show line profiles (vertical) over the center frame before and after correction, where in this scene the line profiles traverse both cloudy and non-cloudy scenes. The 

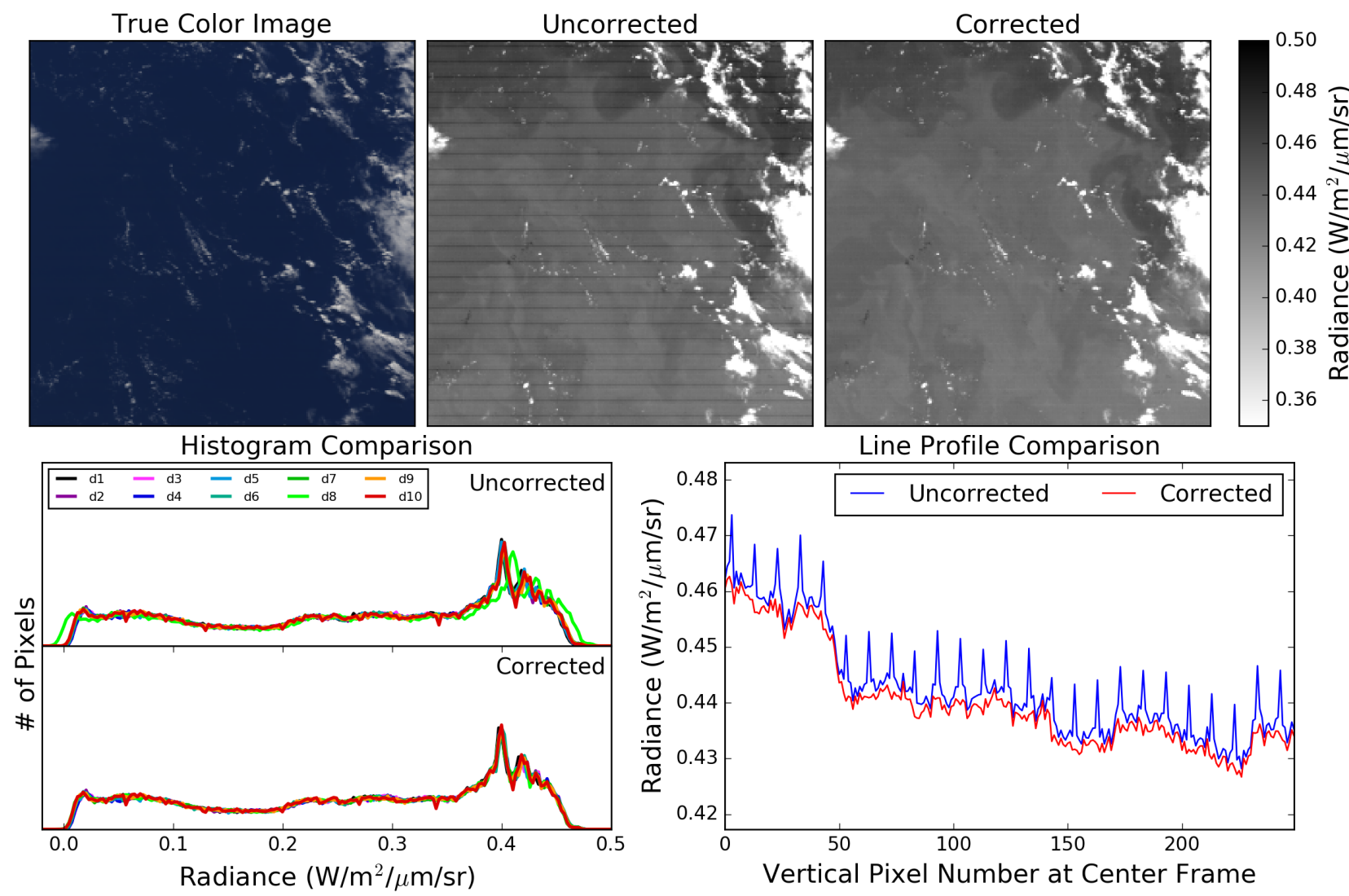

Figure 10. Example correction for band 22 over ocean on June 21, 2016. (top) True color, uncorrected and corrected images. The scale for the band 22 images is truncated to clearly show the striping over the ocean scene in the uncorrected image. (bottom left) A histogram comparison for the entire 5 minute granule containing the selected scene before and after a correction. (bottom right) A vertical line-profile comparison through the center frame of the selected scene.

line profiles clearly show that the contamination of the signal occurs only over the clouds, where spikes can be seen in the uncorrected profile. After correction, we can see that the striping is removed from the cloudy part of the scene while no new striping is introduced in the higher radiance scenes over ocean.

For bands 20, 21 and 25, the crosstalk correction based on the lunar data has little impact. For band 20, when analyzing a wide variety of Earth-view scenes, we do not observe the types of detector differences or image striping seen in bands $22-24$, and therefore it is likely that no correction needs to be made for this band. For band 25, we do observe detector-differences for high-radiance scenes on the order of $2 \%$, which is spread over all of the detectors, and does not have a single out-of-family detector as in the case of bands 22 and 23 for these scenes. The lunar-based correction does little to change these radiance values, as the level of contamination in the lunar images for all of the detectors is small. Therefore, a different approach may be needed in order to correct for these detector differences.

For band 21, some image striping is observed in high-radiance scenes over desert for detector 1, which is likely due to the contamination from band 23 detector 10, and over ocean scenes for detector 9 . However, in this case of detector 1, we find that the performance of the lunar-based correction is insufficient to consistently correct this data. The striping is on the order $20 \%$, and approximately half of the contamination is removed using the coefficient derived from the lunar data. The striping can be more effectively removed by increasing the crosstalk coefficient from band 23 detector 10 sending by a factor of 2 , but this type of increase is not justified by our lunar observation data. Therefore, another method for deriving a correction may be necessary. For the detector 9 striping over ocean scenes, the correction does provide a noticeable improvement to the image striping 

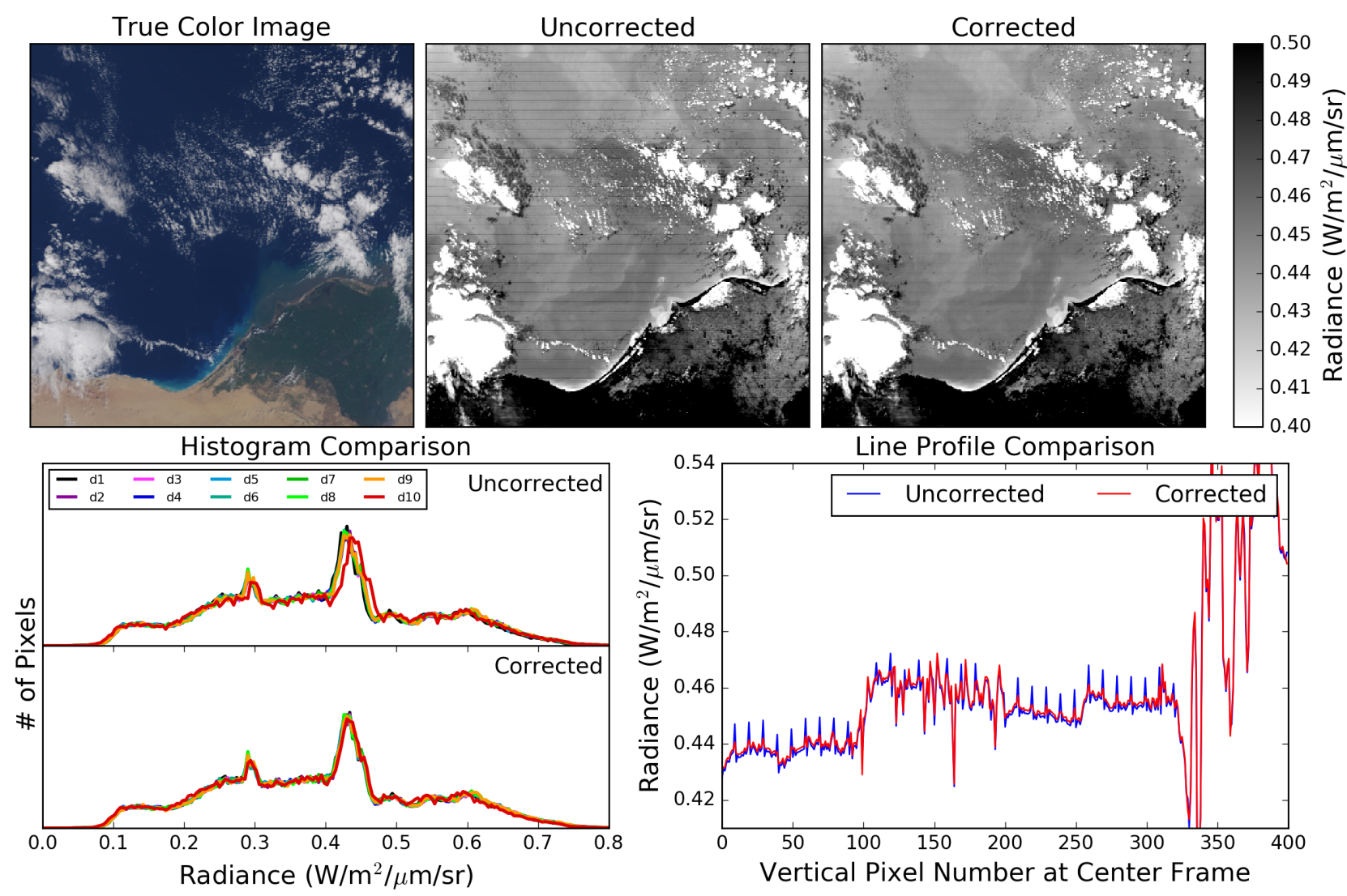

Figure 11. Example correction for band 23 over ocean on February 1, 2008. (top) True color, uncorrected and corrected images. The scale for the band 23 images is truncated to clearly show the striping over the water scene in the uncorrected image. (bottom left) A histogram comparison for the entire 5 minute granule containing the selected scene before and after a correction. (bottom right) A vertical line-profile comparison through the center frame of the selected scene.

for these scenes. However, the band 21 signal is typically noisy over these ocean scenes. In general, band 21 is a low signal band that is used to observe high-radiance fire scenes, and because of this does not use non-linear gain coefficients to produce the L1B product, as the BB warm-up/cool-down cycles do not reach the same scene temperatures as those over fire. So, some special considerations may need to be made in order to appropriately correct the data for this band. However, this band is typically only used as a backup to band 22 in higher level MODIS data products that involve fire detection, and is considered a low-priority for correction.

A summary of the impact of electronic crosstalk on the MWIR bands of Terra MODIS and the effectiveness of our correction is presented in Table 2. Overall, using our derived crosstalk coefficients provided a good correction to the detectors with the most significant levels of contamination. The combination of the coefficients derived from the lunar observations and the KL method for band 26 sending provides the best set of correction coefficients over the widest range of scenes. However, for some bands, more work needs to be done to improve the correction and may necessitate a new method for analyzing the crosstalk contamination. While the correction results in improved L1B image quality, an analysis of the impact of the correction on higher level MODIS products needs to be performed before this correction can be implemented in the official Level-1B algorithm.

\section{SUMMARY AND FUTURE WORK}

We have analyzed the crosstalk contamination for the MWIR bands, 20 - 25, in Terra MODIS using both lunar observations and Earth-view imagery. Using the lunar observations, we were able to derive correction coefficients for each band and detector throughout the mission. For most detectors, the impact of the crosstalk contamination 


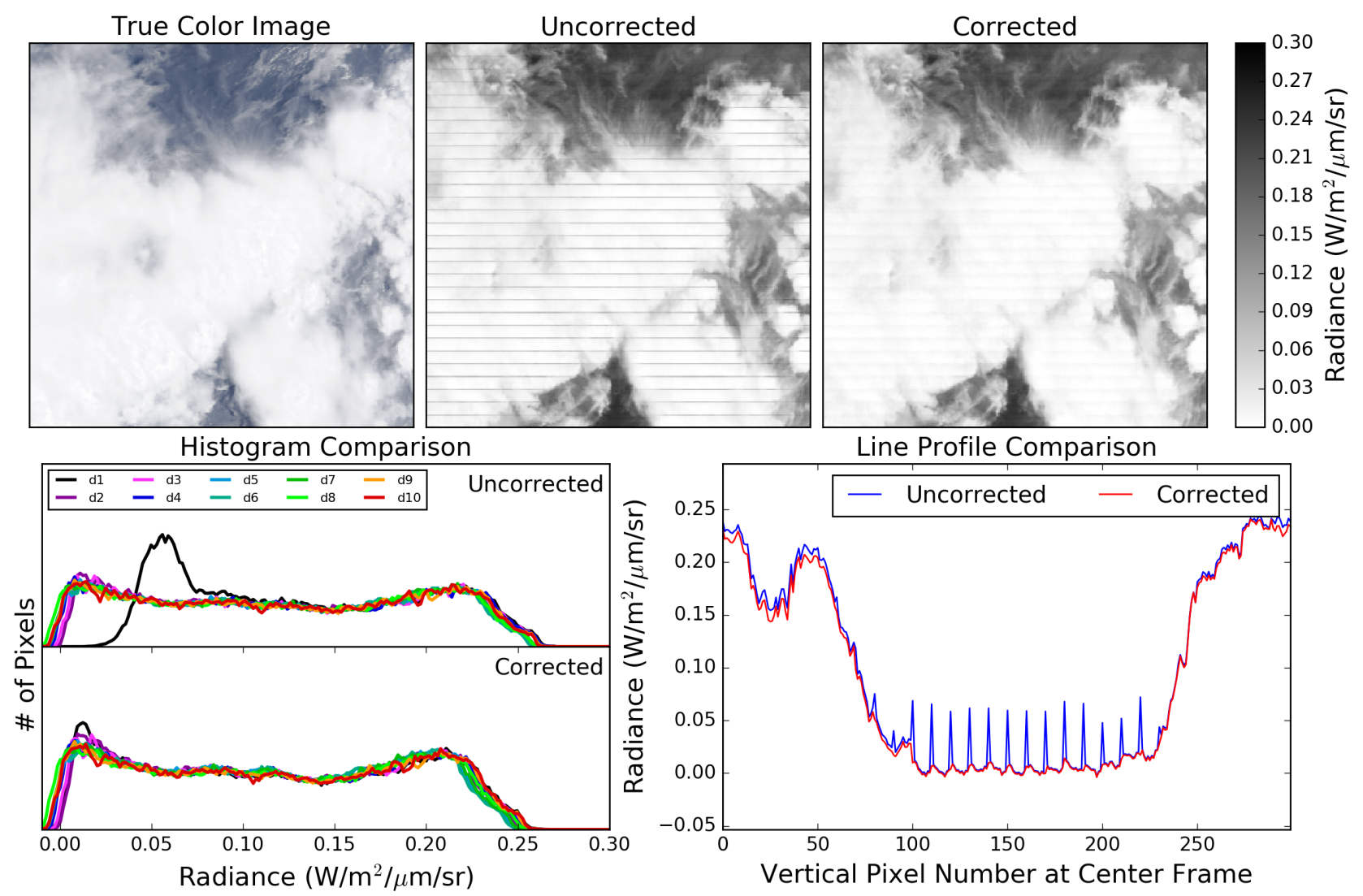

Figure 12. Example correction for band 24 for an ice cloud scene over ocean on February 1, 2008. (top) True color, uncorrected and corrected images. (bottom left) A histogram comparison for the entire 5 minute granule containing the selected scene before and after a correction. (bottom right) A vertical line-profile comparison through the center frame of the selected scene.

Table 2. A summary of the impact of the crosstalk contamination on the Terra MODIS MWIR bands and the effectiveness of the correction.

\begin{tabular}{ccc}
\hline \hline Band & Contamination & Correction \\
\hline 20 & Negligible & No correction needed \\
21 & Detectors 1 and 9 striping for warm water and desert scenes & Limited improvement \\
22 & Detector 8 striping for ice cloud scenes and warm water scenes & Successful correction \\
23 & Detector 10 striping for ice cloud scenes and warm water scenes & Successful correction \\
24 & Detector 1 striping for ice cloud scenes & Successful correction \\
25 & Detector differences for high-radiance scenes & No improvement \\
\hline
\end{tabular}

was negligible in the L1B product. However, for bands $22-24$, we found that a single detector in each band respectively showed out-of-family behavior that was related to the crosstalk contamination. For high-radiance scenes, the correction coefficients that were derived from lunar observations were adequate. However, at low radiance, particularly over ice clouds, the contamination from band 26 sending was not appropriately modeled by the lunar data. In this work, we developed an alternative method using the histograms of Earth-view radiance retrieval in a Kullback-Leibler minimization routine in order to derive the appropriate correction coefficients. Using the combined coefficients from the Moon and KL data significantly improved the image quality over a wide variety of scenes. 
While this method works well for bands $22-24$, for bands 21 and 25 , there are still some detector differences observed in the L1B images that are not corrected by our current approach to the crosstalk correction. While it is possible that these differences are associated with a separate effect, it is prudent to explore other methods for a crosstalk correction using sources other than the moon. This is made clear by the need for the KL-based correction for band 26 sending to bands $22-24$. While the KL method works well for a single, out-of-family detector, applying this method to all of the detectors in a band simultaneously will be difficult without a reference signal. However, this Earth-view based approach might be adapted using other comparison methods to provide a better correction. This possibility will be explored in the future.

\section{ACKNOWLEDGMENTS}

The authors would like to thank Dr. Aisheng Wu for his comments and review of this work. The authors would also like to acknowledge all of the current and former MCST members who have contributed to the long history of electronic crosstalk study of the MODIS instrument.

\section{REFERENCES}

[1] Barnes, W. L., Xiong, X., and Salomonson, V. V., "Status of Terra MODIS and Aqua MODIS," Proc. IGARSS 2, 970-972 (2002).

[2] Salomonson, V. V., Barnes, W. L., Xiong, X., Kempler, S., and Masuoka, E., "An overview of Earth Observing System MODIS instrument and associated data systems performance," Proc. IGARSS 2, 1174$1176(2002)$.

[3] Xiong, X., Sun, J., Barnes, W. L., Salomonson, V. V., Esposito, J., Erives, H., and Guenther, B., "Multiyear on-orbit calibration and performance of Terra MODIS reflective solar bands," IEEE Trans. Geosci. Remote Sens. 45(4), 879 (2007).

[4] Xiong, X., Wu, A., Wenny, B. N., Madhavan, S., Wang, Z., Li, Y., Chen, N., Barnes, W. L., and Salomonson, V. V., "Terra and Aqua MODIS thermal emissive bands on-orbit calibration and performance," IEEE Trans. Geosci. Remote Sens. 53(10), 5709 (2015).

[5] Link, D., Wang, Z., and Xiong, X., "Status of MODIS spatial and spectral characterization and performance," Proc. SPIE 9881, 98811G-1 (2016).

[6] Chen, H., Xiong, X., Angal, A., Geng, X., and Wu, A., "Alternative method of on-orbit response-versusscan-angle characterization for MODIS reflective solar bands," J. Appl. Remote Sens. 10(2), 024004 (2016).

[7] Sun, J., Xiong, X., Angal, A., Chen, H., Wu, A., and Geng, X., "Time-dependent response versus scan angle for MODIS reflective solar bands," IEEE Trans. Geosci. Remote Sens. 52(6), 3159-3174 (2014).

[8] Sun, J., Madhavan, S., Xiong, X., and Wang, M., "Electronic crosstalk correction for terra long wave infrared photovoltaic bands," Proc. SPIE 9264, 926412 (2014).

[9] Sun, J., Madhavan, S., and Wang, M., "Investigation and mitigation of the crosstalk effect in Terra MODIS band 30," Remote Sens. 8(3), 249 (2016).

[10] Sun, J. and Wang, M., "Electronic crosstalk in Aqua MODIS long-wave infrared photovoltaic bands," Remote Sens. 8(10), 806 (2016).

[11] Keller, G. R., Wang, Z., Wu, A., and Xiong, X., "Aqua MODIS band 24 crosstalk striping," IEEE Geosci. Remote Sens. Lett. 14(4), 475 (2017).

[12] Wilson, T., Wu, A., Shrestha, A., Geng, X., Wang, Z., Moeller, C., Frey, R., and Xiong, X., "Development and implementation of an electronic crosstalk correction for bands 27-30 in Terra MODIS collection 6," Remote Sens. 9(6), 569 (2017).

[13] Sun, J., Xiong, X., Barnes, W., and Guenther, B., "MODIS reflective solar bands on-orbit lunar calibration," IEEE Trans. Geosci. Remote Sens. 45(7), 2383 (2007).

[14] Menzel, P., Frey, R., and Baum, B., "Cloud top properties and cloud phase algorithm theoretical basis document (MOD 06, Collection 6)." http://modis-atmos.gsfc.nasa.gov/_docs/MOD06_ATBD_2015_05_ 01.pdf (May 2015).

[15] Ackerman, S., Frey, R., Strabala, K., Liu, Y., Gumley, L., Baum, B., and Menzel, P., "Discriminating clear sky from cloud with MODIS, algorithm theoretical basis document (MOD 35, Collection 6)." http: //modis-atmos.gsfc.nasa.gov/_docs/MOD35_ATBD_Collection6.pdf (October 2010).

[16] Kullback, S., [Information Theory and Statistics], John Wiley \& Sons (1959). 